\title{
Nanoscale Structure-Property Relationship in Amorphous Hydrogenated Boron Carbide for Low- $k$ Dielectric Applications
}

\author{
Soohyun Im $^{1}$, Michelle M. Paquette ${ }^{2}$, Mohammed Belhadj-Larbi ${ }^{2}$, Paul Rulis ${ }^{2}$, Ridwan Sakidja ${ }^{3}$, and \\ Jinwoo Hwang ${ }^{1}$ \\ 1. Department of Materials Science and Engineering, The Ohio State University, Columbus, OH 43212 \\ 2. Department of Physics and Astronomy, University of Missouri-Kansas City, Kansas City, MO 64110 \\ 3. Physics, Astronomy, and Materials Science, Missouri State University, Springfield, MO 65897
}

One of the main challenges in scaling of future integrated circuits is the need for new low dielectric constant $($ low- $k$ ) materials that can substantially reduce the resistance-capacitance delay at the system level. The new low- $k$ material must also overcome the fatal falloff in the mechanical properties, known as the low- $k$ death curve, that is typically associated with the low density of the material required to achieve low $k$ values [1]. Amorphous hydrogenated boron carbide (a-BC:H) combines low $k$ with other advantages, including chemical, thermal, electrical, and mechanical reliability, and therefore has recently gained attention as a high-performing low- $k$ material alternative to conventional $\mathrm{SiO}_{2}$ and SiOC:H/SiCO:H. Our a-BC:H films are synthesized using plasma-enhanced chemical vapor deposition of molecular ortho-carborane, which converts into an amorphous polymer upon deposition [2]. The method enables a high degree of variation in structure and composition $\left(\mathrm{a}-\mathrm{B}_{x} \mathrm{C}: \mathrm{H}_{y}\right)$, which allows for tuning the properties of the material by controlling its nanoscale structure. For example, controlling the degree of medium range order $(\mathrm{MRO})$ arising from the connection or gathering of icosahedral $\mathrm{B}_{x} \mathrm{C}$ orthocarborane clusters (Fig. 1a) can enable tuning the dielectric constant and other important properties of the material with high flexibility. Understanding the MRO structure of a-BC:H is therefore crucial, but the characterization of MRO has been difficult because conventional imaging or diffraction methods are insensitive to such ordering due to its small size and elusive nature.

Here we report the characterization of the nanoscale structure of a-BC:H using fluctuation microscopy to understand the details of MRO and how it relates to important properties of the material. Fluctuation microscopy is based on electron nanodiffraction, and measures the structural fluctuation caused by the nanoscale MRO in disordered structure, providing information on the size, type, degree, and volume fraction of MRO (Fig. 1b and 1c) [3]. It requires a few hundred to a thousand nanodiffraction patterns taken from many different areas of the sample (e.g. Fig. 1d to e), and calculates the intensity variance, $V$, among those diffraction intensities, as a function of the scattering vector magnitude, $k$ (Fig. 2a), which represents the structural fluctuation. For example, Fig. 2a shows the $V(k)$ from high- and low-density a$\mathrm{B}_{x} \mathrm{C}: \mathrm{H}$ samples, which are significantly different from each other. $V(k)$ from a high-density sample shows many peaks, indicating that the sample includes a significant degree of MRO in its structure, while the low-density sample does not. By comparing the experimental $V(k)$ to the simulated $V(k)$ from known atomistic models, such as molecular dynamics (MD) generated models, the details of the nanoscale structural origins of the peaks at $V(k)$ can be analyzed (Fig. 2b). For example, the first peak in $V(k)$ matches the simulated $V(k)$ peak from an MD-generated model that has a clear separation between the super-clustering of the icosahedral clusters. This implies that the peak includes the information on how those superclustered MRO domains are separated from each other. The second peak, on the other hand, matches the simulated $V(k)$ from the known $\mathrm{B}_{4} \mathrm{C}$ crystal (Fig. 2c) [2], suggesting that MRO that resembles that of the $\mathrm{B}_{4} \mathrm{C}$ crystal is abundant in the structure. The detailed relationship between such MRO structure and important electric/mechanical properties will be discussed in this presentation. 


\section{References}

[1] B. J. Nordell et al., Advanced Electronic Materials 2, 7, 1600073 (2016).

[2] M. M. Paquette et al., J. Phys.: Condens. Matter 23, 435002 (2011).

[3] J. Hwang et al., Phys. Rev. Lett. 108, 195505 (2012).
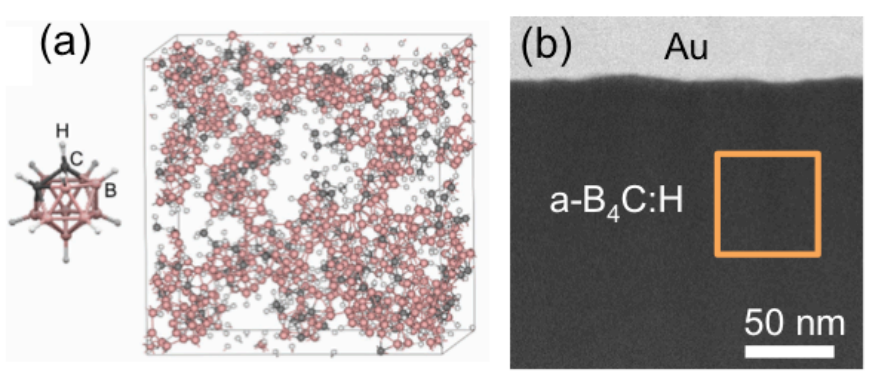

(c)
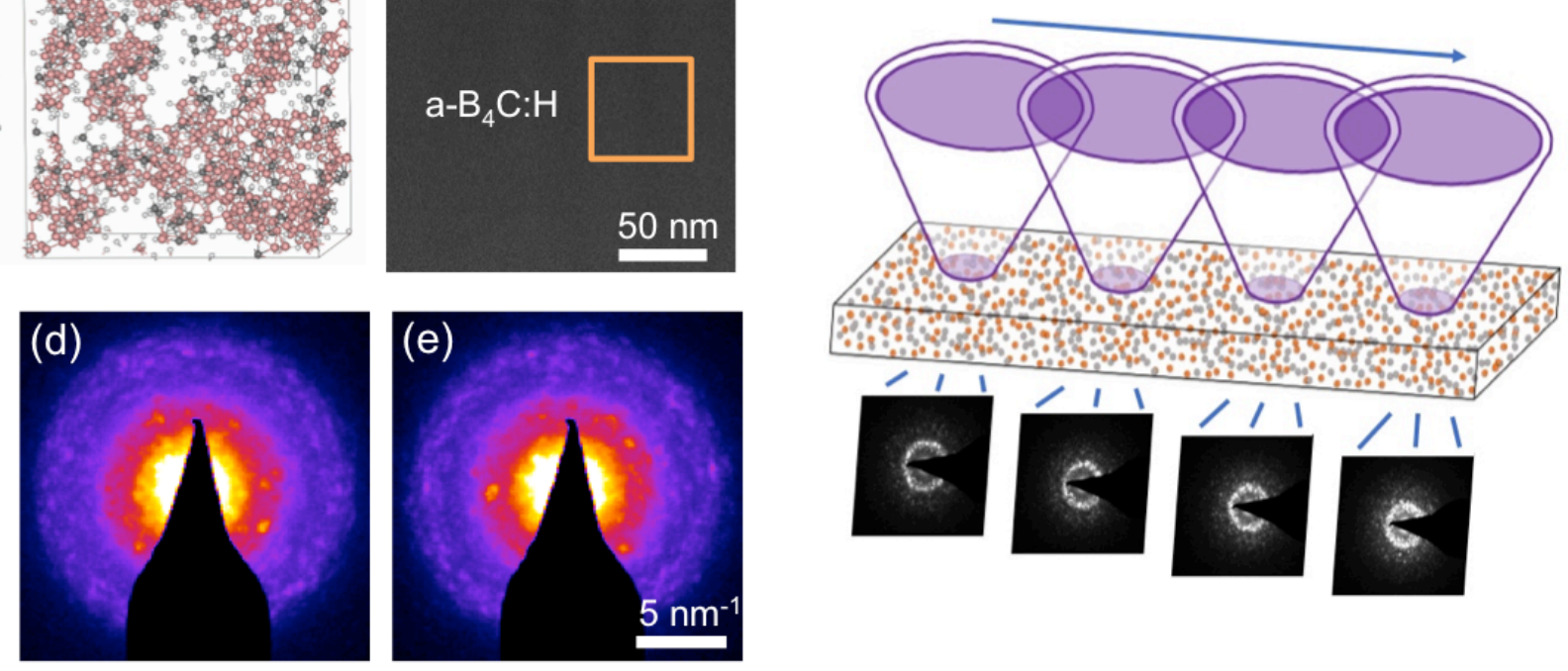

Figure 1. (a) Structure of ortho-carborane icosahedral cluster and their potential gathering (MRO) predicted by a MD simulation [1] (b) STEM Z-contrast image of a-BC:H. (c) Schematic showing nanodiffraction acquisition for fluctuation microscopy. Typically up to ten areas of the sample, such as the one shown in the boxed area in (b), are used, and from each box, nanodiffraction patterns are taken with 10 by 10 grid probe positions. (d and e) Example nano-diffraction patterns showing different speckle patterns, suggesting that the two areas have different MRO structure at the length scale of the probe size $(1.5 \mathrm{~nm})$.

(a)

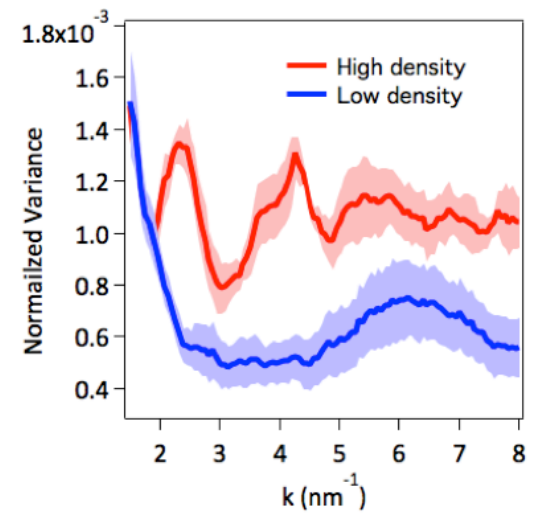

(b)

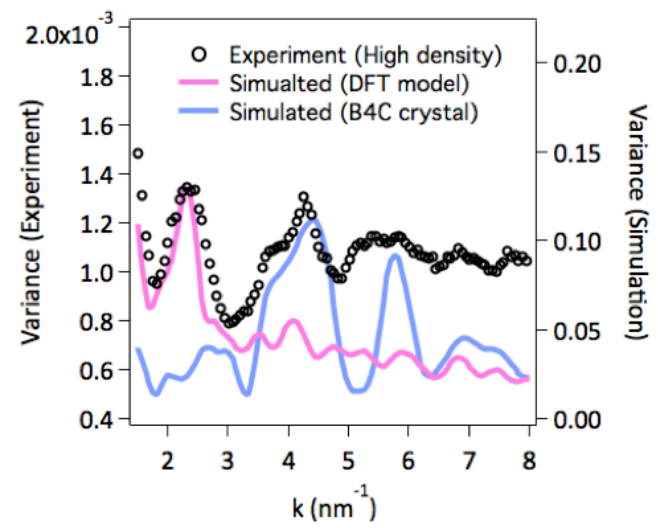

(c)

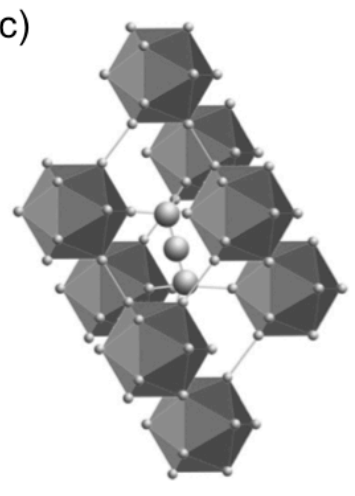

Figure 2. (a) Experimental FEM data, $V(k)$, from high- and low-density a-BC:H; (b) Comparison between the experimental $V(k)$ (high density) and simulated $V(k)$ 's from a DFT-generated and a $\mathrm{B}_{4} \mathrm{C}$ crystal model shown in (c). 